\title{
Collaborative Rough Clustering
}

\author{
Sushmita Mitra ${ }^{1}$, Haider Banka ${ }^{1}$, and Witold Pedrycz ${ }^{2}$ \\ 1 Machine Intelligence Unit, Indian Statistical Institute, \\ Kolkata 700 108, India \\ \{sushmita, hbanka_r\}@isical.ac.in \\ 2 Dept. of Electrical \& Computer Engg., University of Alberta, \\ Edmonton, Canada T6G 2G7 \\ pedrycz@ece.ualberta.ca
}

\begin{abstract}
A novel collaborative clustering is proposed through the use of rough sets. The Davies-Bouldin clustering validity index is extended to the rough framework, to generate the optimal number of clusters during collaboration.
\end{abstract}

Keywords: Rough sets, collaborative clustering, cluster validity.

\section{Introduction}

A cluster is a collection of data objects which are similar to one another within the same cluster but dissimilar to the objects in other clusters. Clustering large data is a mining problem of considerable interest. A recent trend in intelligent system design for large-scale problems is to split the original task into simpler subtasks and use a module for each of the subtasks. It has been shown that by combining the output of several modules in an ensemble, one can improve the generalization ability over that of a single model [1].

Collaborative clustering deals with revealing a structure that is common or similar to a number of subsets [2]. For example, let us consider a large population of data about client information, distributed over multiple databases. An intelligent approach to mine such large volume of information would be to analyze each individual database of sub-population locally and subsequently combine (or collaborate on) the results at a globally abstract level. This also satisfies certain security (or privacy) concerns of clients in not allowing the sharing of individual data (or samples).

Recently various soft computing methodologies have been applied to handle the different challenges posed by data mining [1, involving large heterogeneous datasets and clustering [1]. Fuzzy sets and rough sets have been incorporated in the $c$-means framework to develop the fuzzy $c$-means (FCM) 3] and rough $c$-means (RCM) 4] algorithms. Typically, rough sets [5] are used to model the clusters in terms of upper and lower approximations. Collaborative clustering has been investigated by Pedrycz [2, using the FCM algorithm.

In this article we present a novel collaborative clustering through the use of rough sets. The Davies-Bouldin (DB) index is extended to the rough framework, 
and helps in determining the optimal number of clusters during collaboration. Section 2 provides the basic description of the $c$-means and RCM clustering algorithms, along with the modified DB index. Collaboration in the RCM framework is presented in Section 4. Experimental results are demonstrated in Section 5. Finally Section 6 concludes the article.

\section{Rough Clustering}

The $c$-means algorithm proceeds by partitioning $N$ objects into $c$ nonempty subsets. During each partition, the centroids or means of the clusters are computed. The main steps of the $c$-means algorithm [6] are as follows:

1. Assign initial means $\mathbf{m}_{i}$ (also called centroids).

2. Assign each data object $\mathbf{X}_{k}$ to the cluster $U_{i}$ for the closest mean.

3. Compute new mean for each cluster using

$$
\mathbf{m}_{i}=\frac{\sum_{\mathbf{X}_{k} \in U_{i}} \mathbf{X}_{k}}{\left|c_{i}\right|}
$$

where $\left|c_{i}\right|$ is the number of objects in cluster $U_{i}$.

4. Iterate until criterion function converges, i.e., there are no more new assignments.

The theory of rough sets [5] has recently emerged as another major mathematical tool for managing uncertainty that arises from granularity in the domain of discourse. The intention is to approximate a rough (imprecise) concept in the domain of discourse by a pair of exact concepts, called the lower and upper approximations. These exact concepts are determined by an indiscernibility relation on the domain, which, in turn, may be induced by a given set of attributes ascribed to the objects of the domain. The lower approximation is the set of objects definitely belonging to the vague concept, whereas the upper approximation is the set of objects possibly belonging to the same.

In the rough $c$-means (RCM) algorithm, the concept of $c$-means is extended by viewing each cluster as an interval or rough set [4. A rough set $Y$ is characterized by its lower and upper approximations $\underline{B} Y$ and $\bar{B} Y$ respectively. This permits overlaps between clusters. Here an object $\mathbf{X}_{k}$ can be part of at most one lower approximation. If $\mathbf{X}_{k} \in \underline{B} Y$ of cluster $Y$, then simultaneously $\mathbf{X}_{k} \in \bar{B} Y$. If $\mathbf{X}_{k}$ is not a part of any lower approximation, then it belongs to two or more upper approximations.

Adapting eqn. (1), the centroid $\mathbf{m}_{i}$ of cluster $U_{i}$ is computed as

$$
\mathbf{m}_{i}=\left\{\begin{array}{lr}
w_{l o w} \frac{\sum_{\mathbf{x}_{k} \in \underline{B} U_{i}} \mathbf{x}_{k}}{\left|\underline{B} U_{i}\right|}+w_{u p} \frac{\sum_{\mathbf{x}_{k} \in\left(\bar{B} U_{i}-\underline{B} U_{i}\right)} \mathbf{x}_{k}}{\left|\bar{B} U_{i}-\underline{B} U_{i}\right|} \text { if } \underline{B} U_{i} \neq \emptyset \wedge \bar{B} U_{i}-\underline{B} U_{i} \neq \emptyset \\
\frac{\sum_{\mathbf{x}_{k} \in \underline{B} U_{i}}}{\sum_{k} \underline{B}_{i} \mid} & \text { if } \bar{B} U_{i}-\underline{B} U_{i}=\emptyset \\
\frac{\left.\sum_{\mathbf{x}_{k} \in\left(\bar{B} U_{i}-\underline{B} U_{i}\right.}\right) \mathbf{X}_{k}}{\left|\bar{B} U_{i}-\underline{B} U_{i}\right|} & \text { otherwise, }
\end{array}\right.
$$


where the parameters $w_{\text {low }}$ and $w_{u p}$ correspond to the relative importance of the lower and upper bounds respectively. Here $\left|\underline{B} U_{i}\right|$ indicates the number of patterns in the lower approximation of cluster $U_{i}$, while $\left|\bar{B} U_{i}-\underline{B} U_{i}\right|$ is the number of elements in the rough boundary lying between the two approximations.

The RCM algorithm is outlined as follows.

1. Assign initial means $\mathbf{m}_{i}$ for the $c$ clusters.

2. Assign each data object (pattern point) $\mathbf{X}_{k}$ to the lower approximation $\underline{B} U_{i}$ or upper approximation $\bar{B} U_{i}, \bar{B} U_{j}$ of cluster pairs $U_{i}, U_{j}$ by computing the difference in its distance $d_{i k}-d_{j k}$ from cluster centroid pairs $\mathbf{m}_{i}$ and $\mathbf{m}_{j}$.

3. Let $d_{i k}$ be minimal.

If $d_{j k}-d_{i k}$ is less than some threshold

then $\mathbf{X}_{k} \in \bar{B} U_{i}$ and $\mathbf{X}_{k} \in \bar{B} U_{j}$ and $\mathbf{X}_{k}$ cannot be a member of any lower approximation,

else $\mathbf{X}_{k} \in \underline{B} U_{i}$ such that distance $d_{i k}$ is minimum over the $c$ clusters.

4. Compute new mean for each cluster $U_{i}$ using eqn. (2).

5. Iterate until convergence, i.e., there are no more new assignments.

The expression in eqn. (2) boils down to eqn. (1) when the lower approximation is equal to the upper approximation, implying an empty boundary region. It is observed that the performance of the algorithm is dependent on the choice of $w_{\text {low }}, w_{\text {up }}$ and threshold. We allowed $w_{\text {up }}=1-w_{\text {low }}, 0.5<w_{\text {low }}<1$ and $0<$ threshold $<0.5$.

\section{Clustering Validity Index}

The partitive clustering algorithms, described above, require prespecification of the number of clusters. The results are dependent on the choice of $c$. There exist validity indices to evaluate the goodness of clustering, corresponding to a given value of $c$. In this article we compute the optimal number of clusters $c_{0}$ in terms of the Davies-Bouldin cluster validity index [7.

The Davies-Bouldin index (DB) is a function of the ratio of the sum of within-cluster distance to between-cluster separation. Let $\left\{\mathbf{x}_{1}, \ldots, \mathbf{x}_{\left|c_{k}\right|}\right\}$ be a set of patterns lying in a cluster $U_{k}$. Then the average distance between objects within the cluster $U_{k}$ is expressed as $S\left(U_{k}\right)=\frac{\sum_{i, i^{\prime}}|| \mathbf{x}_{i}-\mathbf{x}_{i^{\prime}}||}{\left|c_{k}\right|\left(\left|c_{k}\right|-1\right)}$, where $\mathbf{x}_{i}, \mathbf{x}_{i^{\prime}} \in U_{k}$ and $i \neq i^{\prime}$. The between-cluster separation is defined as $d\left(U_{k}, U_{l}\right)=\frac{\sum_{i, j}|| \mathbf{x}_{i}-\mathbf{x}_{j} \|}{\left|c_{k}\right|\left|c_{l}\right|}$, where $\mathbf{x}_{i} \in U_{k}, \mathbf{x}_{j} \in U_{l}$, such that $k \neq l$. The optimal clustering, for $c=c_{0}$, minimizes

$$
D B=\frac{1}{c} \sum_{i=1}^{c} \max _{j \neq i}\left\{\frac{S\left(U_{i}\right)+S\left(U_{j}\right)}{d\left(U_{i}, U_{j}\right)}\right\},
$$

for $1 \leq i, j \leq c$. Thereby, the within-cluster distance $S\left(U_{i}\right)$ is minimized while the between-cluster separation $d\left(U_{i}, U_{j}\right)$ gets maximized. 
The rough within-cluster distance is formulated as $S_{r}\left(U_{i}\right)=$

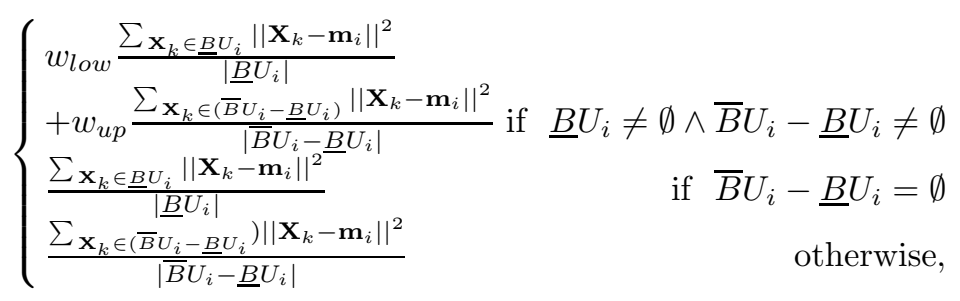

using eqn. (2). Rough DB now becomes

$$
D B_{r}=\frac{1}{c} \sum_{i=1}^{c} \max _{j \neq i}\left\{\frac{S_{r}\left(U_{i}\right)+S_{r}\left(U_{j}\right)}{d\left(U_{i}, U_{j}\right)}\right\} .
$$

\section{Collaborative RCM Clustering}

In this section a collaborative rough $c$-means clustering is proposed, by incorporating collaboration between different partitions or sub-populations. Let a dataset be divided into $P$ sub-populations or modules. Each sub-population is independently clustered to reveal its structure. Collaboration is incorporated by exchanging information between the modules regarding the local partitions, in terms of the collection of prototypes computed within the individual modules. This sort of divide-and-conquer strategy enables efficient mining of large databases. The required communication links are hence at a higher level of abstraction, thereby representing information granules (rough clusters) in terms of their prototypes. There exist two phases in the algorithm.

- Generation of RCM clusters within the modules, without collaboration. Here we employ $0.5<w_{\text {low }}<1$, thereby providing more importance to samples lying within the lower approximation of clusters while computing their prototypes locally.

- Collaborative RCM between the clusters, computed locally for each module of the large dataset. Now we use $0<w_{\text {low }}<0.5$ (we chose $w_{\text {low }}=1-$ $\left.w_{\text {low }}\right)$, with a lower value providing higher precedence to samples lying in the boundary region of overlapping clusters. Here a cluster $U_{i}$ may be merged with an overlapping cluster $U_{j}$

$$
\text { if } \quad\left|\underline{B} U_{i}\right| \leq\left|\bar{B} U_{i}-\underline{B} U_{i}\right|
$$

is maximum for all $i=1, \ldots, c$ and $d\left(\mathbf{m}_{i}, \mathbf{m}_{j}\right)$ is minimum for $j \neq i$.

Let there be $c_{1}$ and $c_{2}$ clusters, generated by RCM, in a pair of modules $(P=2)$ under consideration. During collaboration, we begin with $c_{1}+c_{2}$ cluster prototypes and merge using eqn. (6). 


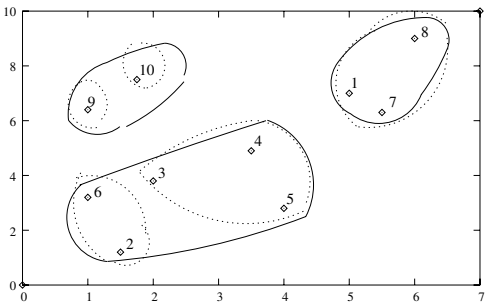

(a)

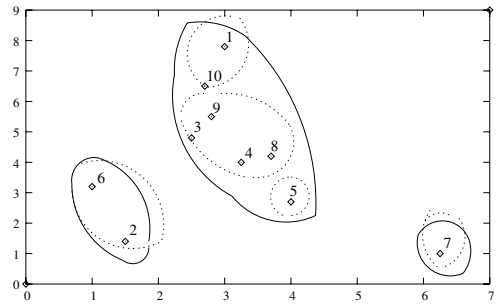

(b)

Fig. 1. Collaborative rough clustering on synthetic dataset for (a) Module A and (b) Module B, with RCM

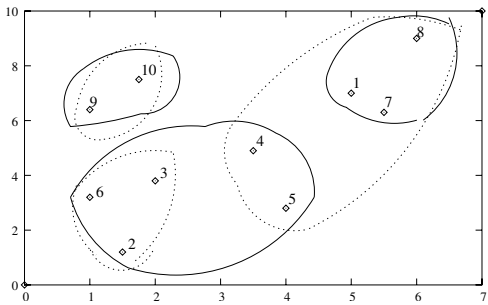

(a)

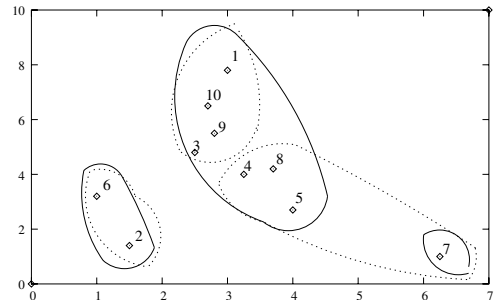

(b)

Fig. 2. Collaborative fuzzy clustering on synthetic dataset for (a) Module A and (b) Module B, with FCM

Table 1. Sample comparative performance of collaborative RCM on synthetic data

\begin{tabular}{|c|c|c|c|c|c|c|}
\hline \multirow[b]{2}{*}{ Module } & \multicolumn{3}{|c|}{ Before collaboration } & \multicolumn{3}{|c|}{ After collaboration } \\
\hline & Prototypes & $\begin{array}{c}\text { Samples in } \\
\text { lower } \\
\text { approximation }\end{array}$ & $\begin{array}{c}\text { Rough } \\
\text { DB }\end{array}$ & Prototypes & $\begin{array}{c}\text { Samples in } \\
\text { lower } \\
\text { approximation }\end{array}$ & $\begin{array}{c}\text { Rough } \\
\text { DB }\end{array}$ \\
\hline A & $\begin{array}{c}(3.35,6.42) \\
(6.0,9.0) \\
(2.13,2.75)\end{array}$ & $\begin{array}{c}1,4,7,9,10 \\
8 \\
2,3,5,6\end{array}$ & 0.789 & $\begin{array}{c}(1.75,7.5) \\
(5.5,7.43) \\
(3.17,3.83) \\
(1.0,6.4) \\
(1.25,2.2)\end{array}$ & $\begin{array}{c}10 \\
1,7,8 \\
3,4,5 \\
9 \\
2,6 \\
\end{array}$ & 0.732 \\
\hline B & $\begin{array}{l}(6.25,1.0) \\
(3.14,5.07) \\
(1.25,2.3)\end{array}$ & $\begin{array}{c}7 \\
1,3,4,5,8,9,10 \\
2,6\end{array}$ & 0.567 & $\begin{array}{c}(2.85,7.15) \\
(4.0,2.7) \\
(6.25,1.0) \\
(3.06,4.63) \\
(1.25,2.3)\end{array}$ & $\begin{array}{c}1,10 \\
5 \\
7 \\
3,4,8,9 \\
2,6\end{array}$ & 0.441 \\
\hline
\end{tabular}

\section{Results}

Results are provided on a two-dimensional synthetic dataset [2, using RCM and FCM, as shown in Fig. 1 and Fig. 2 respectively. There are two modules (A and B) corresponding to ten samples each, partitioned into three clusters. Sample 
results using threshold $=0.2, w_{\text {low }}=0.9$ for $\mathrm{RCM}$, and $w_{\text {low }}=1-0.9=0.1$ for collaborative RCM, are provided in Table 1. It is observed that the Rough DB decreases for both modules after collaboration. Figs. 1)(a) and (b) indicate the clustering before (solid line) and after (dotted line) collaboration, using modules $\mathrm{A}$ and $\mathrm{B}$ respectively with $\mathrm{RCM}$.

\section{Conclusion}

Collaborative clustering is a promising approach towards modeling agent-based systems. While handling large data in this framework, each intelligent agent may concentrate on information discovery (or clustering) within a module. These agents can communicate with each other at the cluster interface, using appropriate protocol, their cluster profiles represented in terms of the centroids.

In this article, we have presented a novel collaborative clustering using the RCM algorithm. The Davies-Bouldin clustering validity index is modified, by incorporating rough concepts, to determine optimal clustering during collaboration.

\section{Acknowledgement}

This work is partly supported by CSIR (No. 22(0346)/02/EMR-II),New Delhi.

\section{References}

1. Mitra, S., Acharya, T.: Data Mining: Multimedia, Soft Computing, and Bioinformatics. John Wiley, New York (2003)

2. Pedrycz, W.: Collaborative fuzzy clustering. Pattern Recognition Letters 23 (2002) $1675-1686$

3. Bezdek, J.C.: Pattern Recognition with Fuzzy Objective Function Algorithms. Plenum Press, New York (1981)

4. Lingras, P., West, C.: Interval set clustering of Web users with rough $k$-means. Technical Report No. 2002-002, Dept. of Mathematics and Computer Science, St. Mary's University, Halifax, Canada (2002)

5. Pawlak, Z.: Rough Sets, Theoretical Aspects of Reasoning about Data. Kluwer Academic, Dordrecht (1991)

6. Tou, J.T., Gonzalez, R.C.: Pattern Recognition Principles. Addison-Wesley, London (1974)

7. Bezdek, J.C., Pal, N.R.: Some new indexes for cluster validity. IEEE Transactions on Systems, Man, and Cybernetics, Part-B 28 (1998) 301-315 